\title{
Shedding some light on the use of mesh in gastroesophageal junction surgery
}

\author{
L Fei , F Moccia, M Cimmino, V Trapani, G Romano \\ From de Senectute: Age and Health Forum \\ Catanzaro, Italy. 5-7 December 2009
}

\section{Background}

Postoperative complications such as failure or disruption of the crura repair and intrathoracic migration of the wrap are the most common anatomic reasons for the failure of Laparoscopic Nissen Fundoplication.

The authors hypothesized that ultrastructural illness may be implicated in this recurrence. The aim of this study was to investigate the presence of changes at esophageal hiatal area in patients with and without $\mathrm{HH}$ and to shed some light on the use of mesh in this surgery.

\section{Materials and methods}

We enrolled 33 patients affected by simultaneous hiatal hernia and GERD (HH group) and 14 with cholelithiasis and one with a symptomatic cyst of the spleen (control group). All specimens from phrenoesophageal membrane and diaphragmatic crura were processed and analyzed by transmission electron microscopy. The grading of the identified ultrastructural muscular lesions ranged from a low severity degree (type 1) to a high severity degree (type 4), as detailed in Table 1.

\section{Results}

Ultrastructural muscular lesions were present in a mix of combinations in $94 \%(31 / 33)$ of the patients affected by $\mathrm{HH}$; they showed at least one or more than one of the

Table 1 Grading of the Electron Microscope Pillar

\section{Changes}

\begin{tabular}{ll}
\hline Grade & Ultrastructural findings \\
II & Dilation of the intermyofibrillar spaces \\
III & Swelling of sarcotubular structures \\
IV & Extended disruption-degeneration of the muscle architecture \\
\hline
\end{tabular}

Department of Clinical and Experimental Medicine and Surgery "F. MagrassiA. Lanzara". Unit of General Surgery and Digestive Physiopathology, Second University of Naples, 80131 Naples, Italy

main types of electron microscope alterations. These findings were present in each of two pillar samples: types I, II, III, and IV muscular changes have been documented in $45 \%, 39 \%, 51 \%$, and $75 \%$ of the cases, respectively.

\section{Conclusions}

We theorized that the changes of the hiatus may affect the ultrastructure of its sarcolemmal-plasmic components as well as the extracellular matrix of the muscle. This hypothesis was sustained by the observation of high frequency relapses of hiatus hernia after traditional surgical intervention in spite of the lack of an objective and/or instrumental evidence of any alteration affecting the muscle-connective components of the diaphragm in such type of patients. We could deduce that the diaphragmatic crural alterations could influence the outcome of hiatal hernia repair as it occurs for inguinal, ventral, and/or incisional hernias. This concept is supported by the fact that the ultrastructural changes were found only in the muscular tissue, and by the fact that the incidence of severe muscular lesions, as types IV and III, was very high $(75 \%$ and $51 \%$, respectively) in our patients. The use of a prosthetic reinforced hiatoplasty seems to significantly reduce the risk of postoperative disruption of hiatal repair. However, the use of mesh at the hiatus may result in the additional risk of erosion, migration of the mesh into the esophagus or stomach as well as the development of fibrotic strictures or adhesions in the hiatal area over the long term. Future randomized trials are needed to confirm that mesh repair is superior to simple crural closure.

Published: 19 May 2010

doi:10.1186/1471-2318-10-S1-A8

Cite this article as: Fei et al: Shedding some light on the use of mesh in gastroesophageal junction surgery. BMC Geriatrics 2010 10(Suppl 1):A8. 